\title{
Congenital fistula between the aorta and left atrium
}

\author{
PETROS NIHOYANNOPOULOS, RALPH SAPSFORD, CELIA M OAKLEY \\ From the Departments of Medicine (Clinical Cardiology) and Surgery (Cardiovascular), Royal Postgraduate \\ Medical School, Hammersmith Hospital, London
}

SUMMARY A child in whom abnormal vessels connected the descending thoracic aorta and the left atrium is described. This is a previously unreported congenital malformation. Clinically this condition differs from the more common aorto-cardiac fistulas in that the continuous murmur was better heard posteriorly as well as in the right parasternal area. Ligation of the aberrant vessels abolished the murmur.

Aorto-cardiac fistulas are well known. Aneurysms of the sinuses of Valsalva, usually of the right sinus, may rupture into the right ventricle, right atrium, or rarely the left atrium. ${ }^{12}$ The communications are rarely congenital. They rupture either spontaneously or as a result of infection, trauma, or operation involving the aorta or aortic valve. ${ }^{3-5}$

An aorto-left ventricular tunnel is a congenital anomaly in which a defect in the aortic wall provides a tunnel opening on to the left ventricle via the septum bypassing the aortic valve. ${ }^{6}$ Coronary cameral fistulas are usually congenital and caused by an abnormal connection between a main or branch coronary artery and one of the cardiac chambers. ${ }^{7}$

We report a patient in whom there were congenital fistulas between the abnormal branches of the descending thoracic aorta and the left atrium. We found no published reports of similar cases.

\section{Case report}

A four year old Iranian boy was admitted for investigation of a cardiac murmur. He was born after a normal pregnancy and was healthy until he had a "gland" infection at the age of two. Then a heart murmur was discovered.

On examination he was of normal size and build for his age, apart from a precordial bulge caused by an enlarged heart. He was intelligent and cooperative. He was acyanotic with no clinical signs of heart failure. The arterial pulses were regular and

Requests for reprints to Dr Petros Nihoyannopoulos, Department of Medicine (Clinical Cardiology), Hammersmith Hospital, Ducane Road, London W120HS. symmetrical; however, they were of high volume and ill-sustained. Blood pressure was $90 / 40 \mathrm{~mm} \mathrm{Hg}$. The venous pressure was normal. The left ventricle was greatly enlarged and showed a hyperkinetic impulse. A very loud continuous murmur was heard on auscultation. This was best heard posteriorly to the right of the spine at the level of T5 and T6, but it was also heard widely over the back and much more softly anteriorly. There was an apical third heart sound. The electrocardiogram showed sinus rhythm with voltage criteria typical of left ventricular enlargement. Chest $x$ ray showed considerable cardiomegaly and a large aortic knob. The main pulmonary artery was not enlarged and there was no evidence of a left to right shunt.

Cross sectional echocardiography showed situs solitus with normal intracardiac relations. There were no valvar abnormalities. The left ventricle was enlarged and contracting vigorously. The left atrium was also dilated, whereas the right heart chambers were normal. The ventricular septum was intact. There was no evidence of coarctation. The ascending aorta and the horizontal portion of the arch were enlarged and excessively pulsatile as far as the isthmus. At the isthmus a large vessel appeared to emerge from the descending aorta. This was taken to be a ductus arteriosus even though its continuity with the pulmonary artery was not visualised on the echocardiogram. Because the clinical findings were incompatible with a ductus arteriosus, cardiac catheterisation and angiography were performed. There was no pressure gradient across the valves. An aortogram showed a competent aortic valve and no ductus arteriosus. After the arch of the aorta became opacified the left atrium filled promptly through a large channel which appeared to lie posteriorly and parallel to the descending aorta (figs 1 and 2). A 


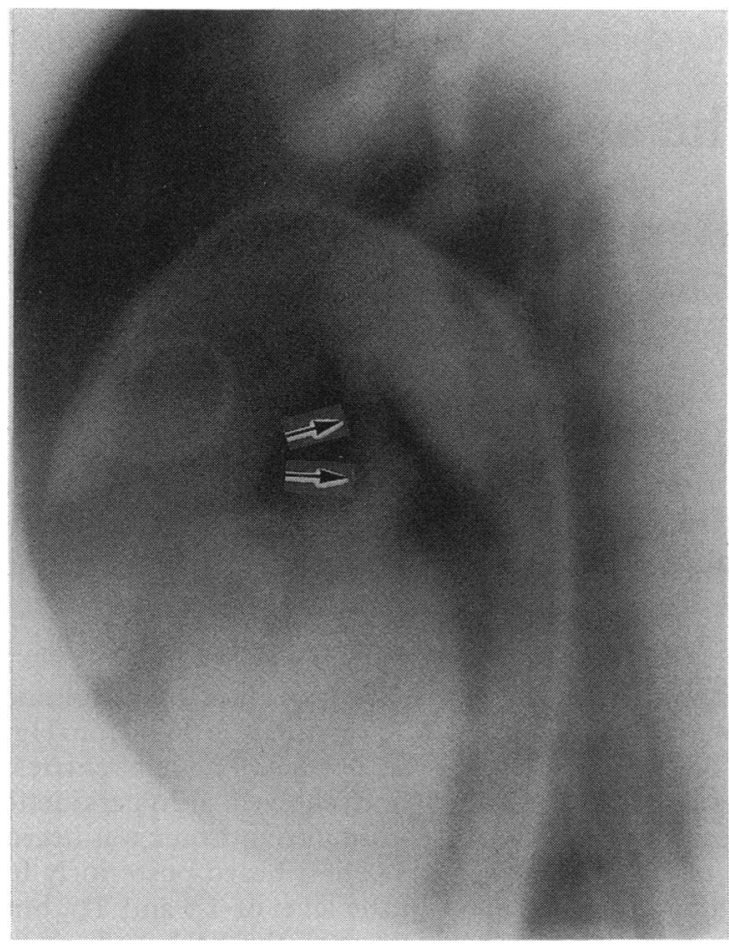

Fig 1 Contrast aortogram in the left anterior oblique projection showing the abnormal vessel connecting the descending thoracic aorta with the left atrium (arrows). Note also the diminishing calibre of the descending thoracic aorta.

fistula between the aorta and left atrium was diagnosed. Because neither the angiogram nor the echocardiogram had indicated the precise connections of this fistula the patient was examined by computed tomography. This showed an anomalous vessel adjacent and to the right of the descending thoracic aorta which appeared in images immediately below the arch of the aorta at a point where the calibre of the aorta abruptly diminished. This structure filled with the contrast to the same degree as the aorta and was of a similar size (fig 3 ).

Laboratory investigations were normal apart from an iron deficiency anaemia (haemoglobin $9.1 \mathrm{~g} / \mathrm{dl}$ ). The tuberculin test was negative.

Operation was performed through a left thoracotomy incision. The aortic arch was large and at the base of a large left subclavian artery there was an abnormal vessel of the same diameter as the left subclavian artery. After about $1.5 \mathrm{~cm}$ this anomalous vessel petered out into a fibrous chord and disappeared into the mediastinum in the region of the oesophagus. About $2 \mathrm{~cm}$ below the origin of the left subclavian artery the descending thoracic aorta rap- idly tapered down to about half the size of the arch? There was a very strong continuous thrill in this. area. After the third part of the arch and the descending thoracic aorta had been fully dissecte $\Phi$ and mobilised, it was possible to see a very large vessel passing directly to the right from the descending thoracic aorta just where it started to narrow and there was a further vessel about half its diameter immediately above it. Because the thrill only disappeared after trial occlusion of both aber? rant vessels, they were evidently in direct commu $\vec{\omega}$ nication. Both vessels were doubly ligated and thi abolished the thrill. The patient's postoperative course was uneventful. Figure 2 is a compositive diagrammatic representation of the angiographic computed tomographic, and operative findings.

\section{Discussion}

Aorto-cardiac communications are not uncommots in children and neonates. The large gradient. between the high pressure aorta and low pressure cardiac chamber causes a high flow for which the heart must compensate to maintain adequate perfusion. ${ }^{8}$ Most congenital aorto-cardiac fistulas com? municate between one of the coronary sinuses, usus ally the right, and the right ventricle, right atriumo and much more rarely the left ventricle. Thes fistulas are often associated with other congenitaf heart lesions. ${ }^{1}$ Jaen et al described a patient with

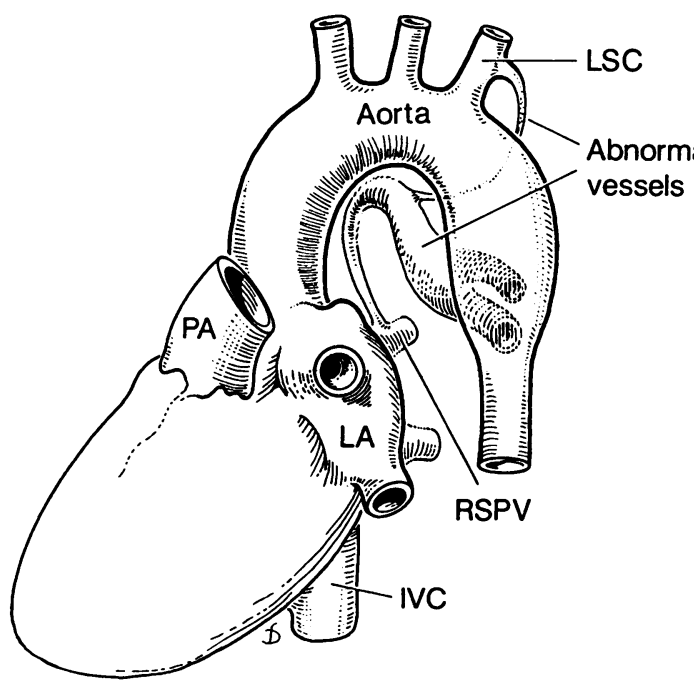

Fig 2 Diagrammatic representation of the aortic arch viewed from the left lateral projection with the pulmonary artery removed for clarity (see text). IVC, inferior vena cava; $L A$, left atrium; $L S C$, left subclavian artery; $P A$, pulmonary artery; $R S P V$, right superior pulmonary vein. . 

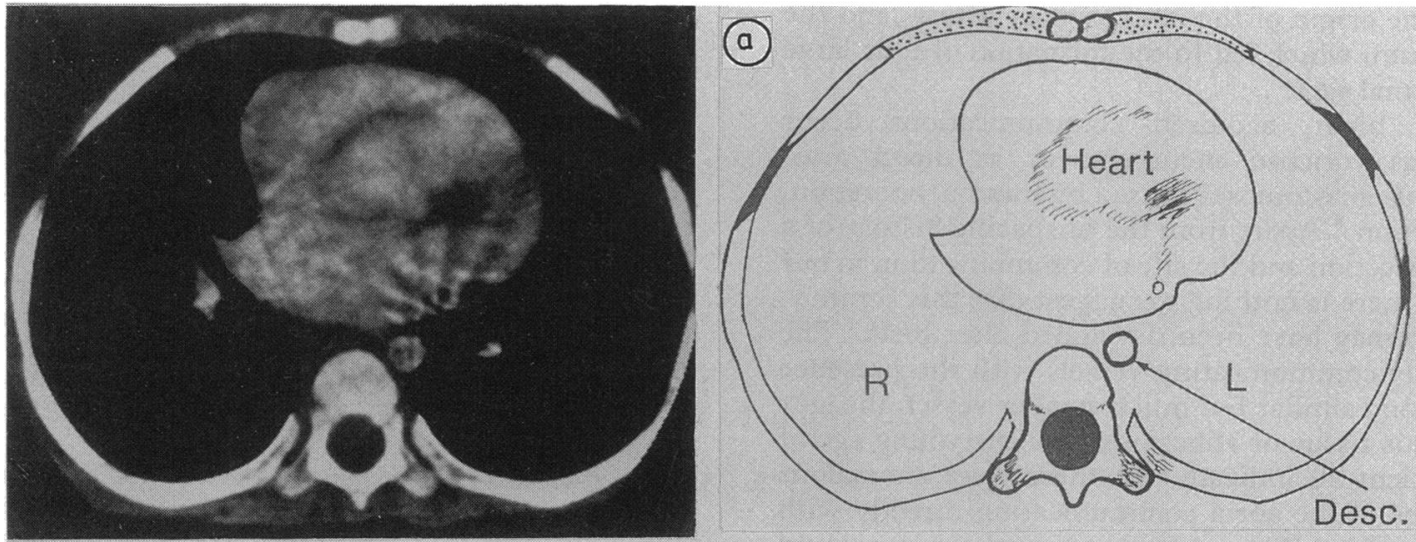

Desc. aorta
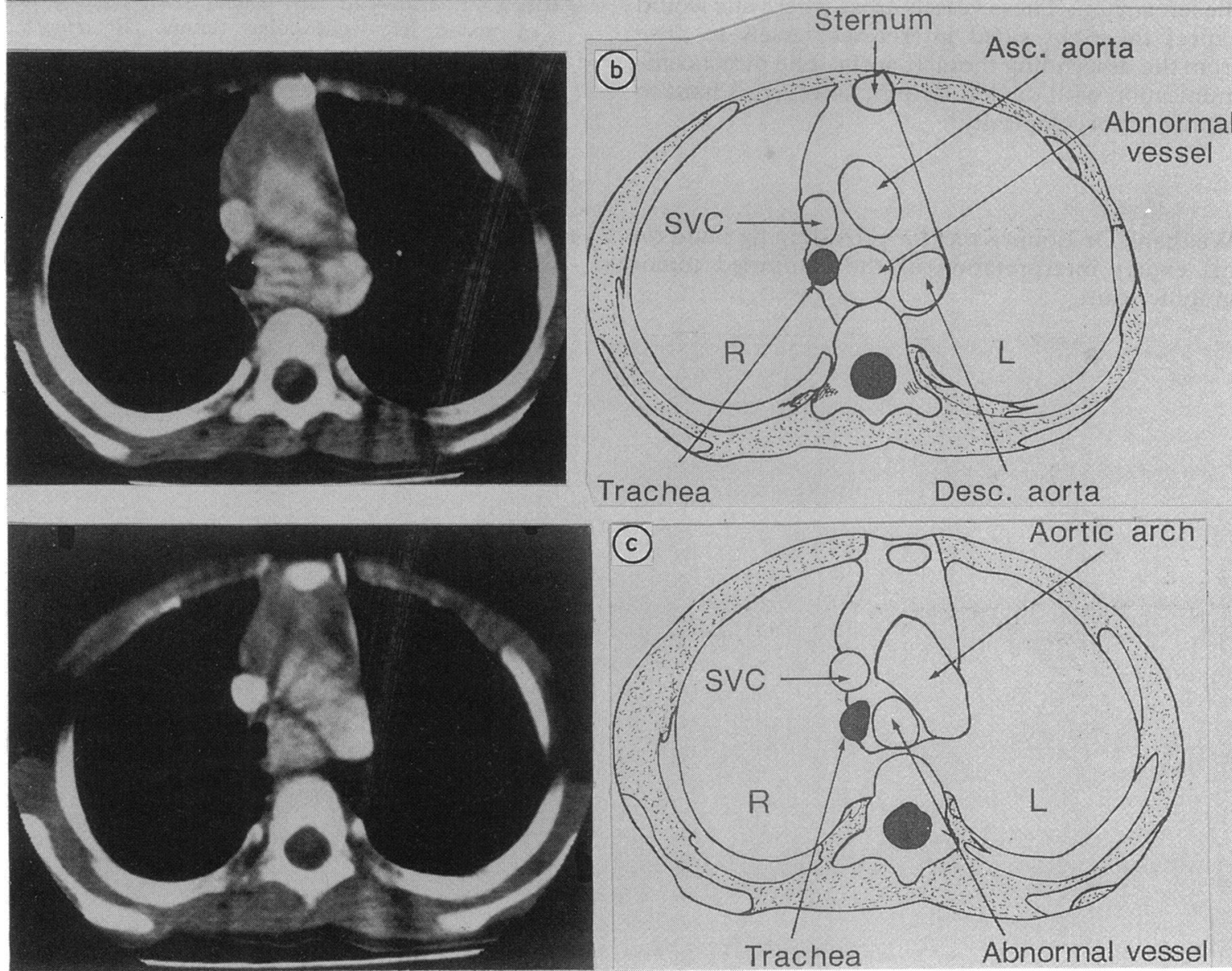

Fig 3 Computed tomographic scan at the level of the heart (a) and great vessels $(b, c)$. After contrast injection $(b, c)$ the abnormal vessel became opacified simultaneously with the ascending and descending aorta $(b)$ and the transverse portion of the $\operatorname{arch}(c)$. The calibre of the descending thoracic aorta at the level of the heart (a) is half the size of the arch (c). 
communication between the ascending aorta, $3 \mathrm{~mm}$ above the origin of the left coronary artery, and the left atrium, which led to the formation of four large aneurysmal sacs. ${ }^{9}$

After birth, acquired communications occur either as varicose aneurysms or as direct arteriovenous anastomoses caused by trauma, operation, or infection. ${ }^{8}$ Apart from the unspecific history of a gland infection and the site of communication in our patient there is nothing to suggest that this communication may have been developed after birth. The smoothly communicating vessels with the presence of a second similar but much smaller vessel, the lack of fibrous tissue or adhesions, and the young age of our patient all indicate that these were anomalous branches of the aorta communicating directly with the left atrium. These vessels arose where one would expect the right sided intercostal vessels to arise from the descending thoracic aorta. The direct communication with the left atrium, however, is hard to explain embryologically.

We thank Dr Louis Kreel for providing fig 3 and for his expert interpretation of the computed tomographic scan.

\section{References}

1 Nowicki ER, Aberdeen E, Friedmann S, Rashkind W Congenital left aortic sinus-left ventricle fistula and review of aortocardiac fistulas. Ann Thorac Sugg 1977;23:378-88.

2 DeBakey ME, Diethrich EB, Liddicoat JE, et Abnormalities of the sinuses of Valsalva. $J$ Thor $\mathbb{S}_{C}$ Cardiovasc Surg 1967;54:312-32.

3 DeSa'Neto A, Padnick MB, Desser KB, Steinhoff NE⿱ Right sinus of Valsalva - right atrial fistula secondagy to non-penetrating chest trauma. Circulation 1979;60:205-9.

4 Datta PK, Vickery $\mathrm{CH}$. Arteriovenous fistula from rug ture of major arterial aneurysms. J R Coll Surg Ediæb 1979;24:165-6.

5 Crean PA, Reid DS, Buchanan J. Aorto-atrial fistula in rheumatoid arthritis. Ann Rheum Dis 1983;42:203-65.

6 Ribeiro P, Bun-Tan LB, Oakley CM. Management of aortic left ventricular tunnel. Br Heart $\bigcirc$ 1985;54:333-6.

7 Urrutia-S CO, Falaschi G, Ott DA, Cooley DA. Sus gical management of 56 patients with congenial coronary artery fistulas. Ann Thorac Surg 1983; 35:300-7.

8 Hudson REB. Cardiovascular pathology. Londọ: Edward Arnold, 1970;3:333-8.

9 Jaen R, Fermin H, Verlarde H, Lairet A, Silva $Q$ Aorto-left atrial fistula. J Cardiovasc Surg (Torin ) 1986;27:355-8. 